\title{
Avian Communities of Alatish National Park, Ethiopia
}

\author{
Takele Tesfahun and Dessalegn Ejigu \\ Department of Biology, College of Science, Bahir Dar University, Bahir Dar, Ethiopia \\ Correspondence should be addressed to Dessalegn Ejigu; dessalegn_ejigu@yahoo.com
}

Received 3 November 2021; Revised 20 December 2021; Accepted 11 January 2022; Published 1 February 2022

Academic Editor: Joao Pedro Barreiros

Copyright (c) 2022 Takele Tesfahun and Dessalegn Ejigu. This is an open access article distributed under the Creative Commons Attribution License, which permits unrestricted use, distribution, and reproduction in any medium, provided the original work is properly cited.

\begin{abstract}
Alatish National Park, which is located in the Amhara National Regional State at the border with the Republic of Sudan, is rich in its avian resources. To study the park's avian communities, the study area was classified based on its vegetation structures into riverine woodland, wooded grassland, woodland, and bamboo woodland. Data were collected from July 2017 to April 2018 using line transect and point count methods. As a result, 132 species of birds belonging to 18 orders and 55 families were identified. The highest species diversities during the wet $\left(\mathrm{H}^{\prime}=3.53\right)$ and dry $\left(\mathrm{H}^{\prime}=3.55\right)$ seasons were recorded in the riverine woodland habitat. Species evenness was also the highest during the wet $(E=0.79)$ and dry $(E=0.77)$ seasons in the riverine woodland habitat. The bamboo woodland habitat harboured the lowest species diversity during the wet $\left(\mathrm{H}^{\prime}=2.81\right)$ and dry $\left(\mathrm{H}^{\prime}=2.45\right)$ seasons. More species similarity $(\mathrm{SI}=0.90)$ was recorded between wooded grassland and bamboo woodland habitats during the dry season. Relative abundance of birds among the different species showed significant differences $\left(F_{131}, 1452=2.94, P<0.05\right)$, and most birds in the area were identified as uncommon species. Forest fire, logging, and overgrazing by cattle have significantly contributed to habitat destruction in the park which might act as the cause for reduction in avian diversity. Therefore, appropriate conservation strategies should be designed and implemented to minimize avian habitat destruction due to various anthropogenic activities within the park.
\end{abstract}

\section{Introduction}

In Ethiopia, there are 881 avian species with 19 endemics, as well as 31 globally threatened and one introduced species [1]. Although most avian species occur in Important Bird Areas (IBAs), these areas represent only $5 \%$ of the country's total area [2]. Amhara National Regional State has been designated as one of the IBAs of the country and comprises $75 \%$ of the Central Ethiopian Highland endemic bird areas [3]. The region has different agroclimatic zones with scenic and biologically fascinating places. Some of the avian species that occur in this region are threatened with extinction, while others such as Abyssinian longclaw (Macronyx flavicollis Rüppell, 1840), Harwood's Francolin (Francolinus harwoodi Blundell and Lovat, 1899), and Wattled ibis (Bostrychia carunculata Rüppell, 1840) are in the IUCN Red Data Book [4].

Species composition in a given area is usually explained by a number of variables that have been found to stress species within a given landscape. These include the area of habitat patch in which the species nests, the amount of habitat within the landscape, degree of disintegration, and vegetation characteristics of the habitat [5]. Environmental heterogeneity in the form of spatial variation in the habitat and local climate can influence the distributions of species [6].

Because they are relatively easy to identify, birds have received considerable attention of humans [7]. They are important for healthy functioning of the ecosystem. Insectivorous birds, for example, control agricultural insect pests [8]. Birds can also act as bioindicators and serve in monitoring the environmental changes [9].

Birds can adapt to every environment that fits their requirements for reproduction and survival [10]. Humans intervene in the natural habitats and greatly decrease the area of naturally functioning ecosystems [11]. Diversity and extent of natural habitats will continue to decline as human population increases and modify the landscape for development $[12,13]$. Forest loss, invasive species, poorly planned infrastructure development, environmental pollution, overexploitation, human induced climate change, and poverty are some of the causes of the decline of birds in the area [5]. 
Effective management of human activities in wildlife protected areas is an important conservation issue as the footprint of human influence continues to expand, and incidental impacts of human activities spread into more areas $[14,15]$. Birds have been mostly useful to assess effects of habitat change because they are easy to observe, and their populations decrease or increase when the landscape is modified by different factors [16]. Birds are indicators and useful models for studying variety of environmental changes $[10,17]$. The objective of this study is, therefore, to study avian community composition in Alatish National Park, Ethiopia, to conserve the park's avifauna in particular and the whole biodiversity resources in general.

\section{Materials and Methods}

2.1. Description of the Study Area. Alatish National Park is located in the West Gondar Administrative Zone, about $1048 \mathrm{~km}$ northwest of Addis Ababa and $311 \mathrm{~km}$ southwest of Gondar town. The Park is bordered in the west and northwest by the Republic of Sudan, in the east and northeast by Quara Woreda, and in the south, southwest, and southeast by Benishangul-Gumuz Regional State. The vegetation of the park is known to act as a "green guard" against the desert expansion from Sudan and Sahel region [18]. The geographic position of the park is within $11^{\circ} 48^{\prime}$ $-12^{\circ} 26^{\prime} \mathrm{N}$ latitude, and $35^{\circ} 16^{\prime}-35^{\circ} 46^{\prime} \mathrm{E}$ longitude (Figure 1). Its topography is relatively flat with an altitude ranging from 354 to 835 m.a.s.l. There are two major rivers, Alatish River and Hyma River, which are sources of water to the park's wildlife and the local people. Moreover, seasonal streams are also available throughout the park during the wet season.

The climate of the park is characterized by two extremes of wet and dry seasons. Effective rain starts in May and peaks in August. The mean monthly temperatures are between 8.9 to $21.6^{\circ} \mathrm{C}$ minimum and 25.8 to $35.2^{\circ} \mathrm{C}$ maximum. The rainfall distribution is unimodal with heavy rainfall from June to September. The highest intensity of rainfall occurs in August which was recorded as $281.2 \mathrm{~mm}$ and the lowest was in February with a record of $0.57 \mathrm{~mm}$ [19].

The vegetation in Alatish National Park is similar to the Sudanian and it is mostly undifferentiated Sudanian and Ethiopian woodland type. The major tree species in the park consists of deciduous trees including Anogeissus leiocarpus, Combretum spp., Boswellia papyrifera, Gardenia ternifolia, Lannea fruticosa, Pterocarpus lucens, Lonchocarpus laxiflorus, Piliostigma thonningii, Terminalia laxiflora, Acacia spp., Stereospermum kunthianum, Sterculia setigera, and Balanites aegyptiaca [20]. The vegetation of the park is classified into riverine woodland, wooded grassland, woodland, and bamboo woodland [20, 21].

2.2. Methods. A preliminary survey to study avian community in the park was carried out for five days from 20 to 25 June 2017. In this survey, topographical features and vegetation cover of the study area were assessed. Additional information about the study area was gathered from scouts of the park and the local people living around the park. Based on the available information, actual field work was carried out from July 2017 to April 2018 comprising the wet and dry seasons.

The study area was classified based on vegetation structure and composition. As a result, four habitat types riverine woodland, wooded grassland, woodland, and bamboo woodland were identified [20]. Data were collected using both line transect and point count methods [22]. Point sampling was used for habitats where bird detectability is limited [23]. Data were recorded by distributing points in the given habitat on a random basis. Transect lines and point count stations were laid on randomly selected blocks.

Data were collected following $[23,24]$. Sampling blocks were established in each of the four vegetation types. The number of blocks was determined according to the size of the area and type of vegetation structure within the park. Based on topographic map and preliminary survey, 19 blocks were identified. The blocks were $6 \times 6 \mathrm{~km}$ area covering of $36 \mathrm{~km}^{2}$. Twenty-six transect lines and eighteen point count stations were sampled at random. The length of each transect line was determined by openness of the habitat [22]. The distance between the consecutive line transects was a minimum of $0.6 \mathrm{~km}$. Birds observed within $100 \mathrm{~m}$ on either side of the transect line were recorded [23].

Point count method was undertaken from a fixed location within the sample unit of radius of $25 \mathrm{~m}$ and with a fixed time interval of 10 minutes [25]. The survey was conducted along a series of point counting stations by counting the number of birds detected [26, 27]. To avoid double counting, routes were spaced as adopted from [28]. In each block, nine-point count stations were identified, and in each point count station, a minimum distance of 150-200 m was maintained using Global Positioning System to avoid double counting [22]. The point counting stations and the width of the transects were set based on the birds' detectability [29].

Along the randomly selected line transects and point count stations, data on avian species diversity, relative abundance, and habitat association were collected for five days per week, and thus a total of 30 days during the wet season and 30 days during the dry season were used. The wet season data were collected during July-August 2017, and data for the dry season were obtained during March-April 2018. Data collections were conducted from $06: 00$ a.m. to 9:30 a.m. and from $03: 00$ p.m. to $06: 00$ p.m. when birds were more active. The speed of walking on the routes depends on the number of birds present and rate of difficulties in recording them. As a result, speeds of $2 \mathrm{~km} \mathrm{~h}^{-1}$ in open habitats and $1 \mathrm{~km} \mathrm{~h}^{-1}$ in dense habitats were used [23]. For identification of bird species, morphological features such as plumage pattern, size, shape, and colour of birds were used as important parameters [30]. Birds were identified using binoculars as well as naked eyes. Photographs of birds were also taken to confirm the identification of some species and birds were identified to the species level based on the field guides $[31,32]$.

2.3. Data Analysis. One-way ANOVA was used to analyze the effect of season and habitat on diversity and relative abundance of birds. Data for the community structure of avian 


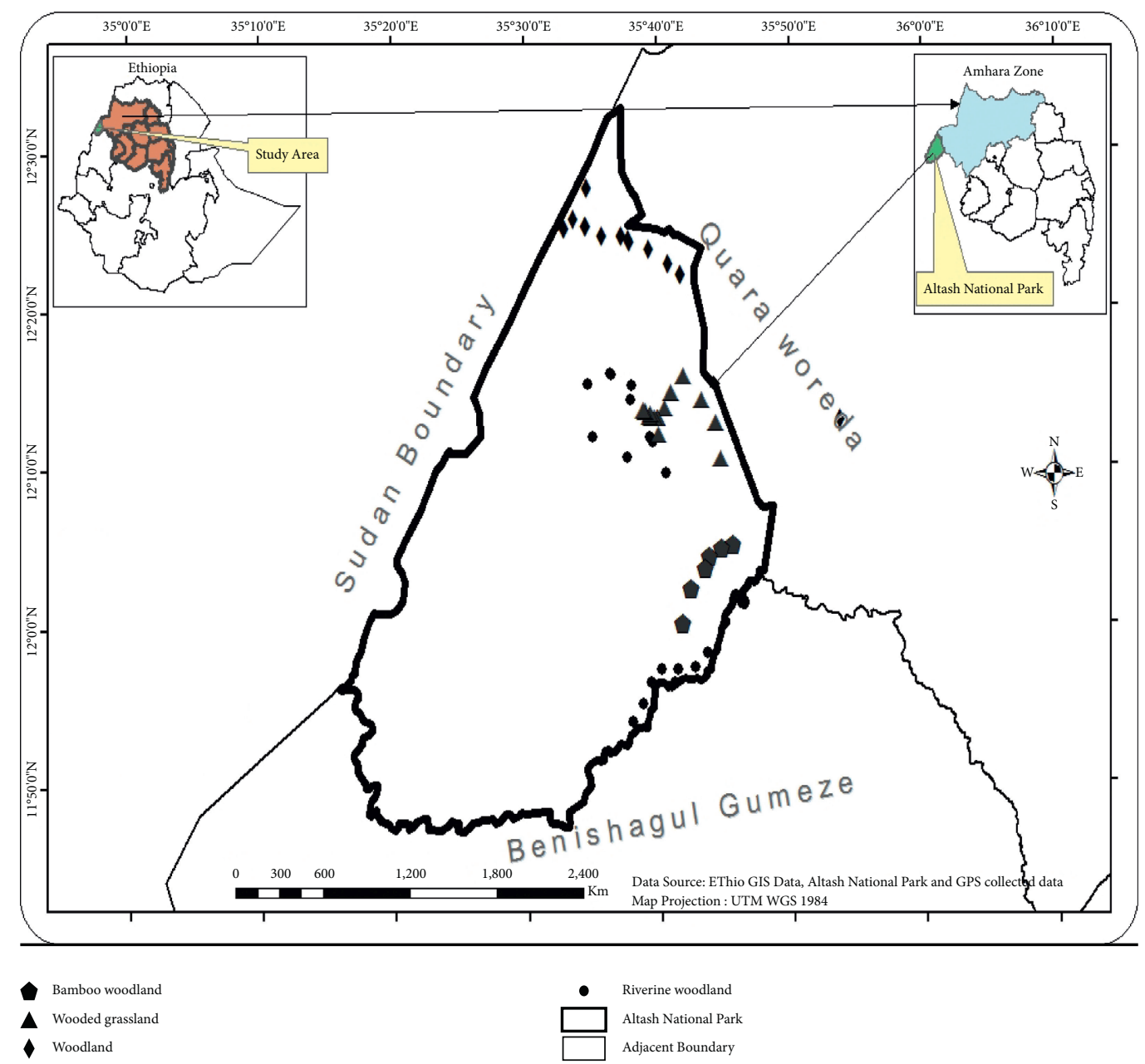

Figure 1: Location map of the study area.

species were analyzed using Shannon-Wiener Diversity Index $\left(\mathrm{H}^{\prime}\right)$ and Simpson's Index of Diversity. Relative abundance of avian species was determined using encounter rates that give scales of abundance as rare, uncommon, frequent, common, and abundant. The abundance category was $<0.1$ (rare), $0.1-2.0$ (uncommon), 2.1-10.0 (frequent), 10.1-40.0 (common), and $40+$ (abundant) [21]. Data analysis was carried out using SAS (version 9.4) software, and Tukey's HSD test at $p=0.05$ was used to test significant differences.

\section{Results}

3.1. Species Composition. A total of 132 avian species belonging to 18 orders and 55 families were identified during the study period, and among these only 56 species were identified as resident birds. A total of 105 species of birds were recorded during the wet season and 86 during the dry season. However, 59 species of birds were recorded during both the wet and dry seasons, 46 species only during the wet season, and 27 species only during the dry season.

African citril (Crithagra citrinelloides Rüppell, 1840) is found only in Ethiopia, Eritrea, and western Kenya, and white-collared pigeon (Columba albitorques Rüppell, 1837) is endemic to both Ethiopia and Eritrea. Black kite (Milvus migrans Boddaert, 1783), cattle egret (Bubulcus ibis Linnaeus, 1758), glossy ibis (Plegadis falcinellus Linnaeus, 1766), Levaillant's cuckoo (Clamator levaillantii Swainson, 1829), great white egret (Ardea alba Linnaeus, 1758), and sacred ibis (Threskiornis aethiopicus Latham, 1790) are African migrants. Grey wagtail (Motacilla cinerea Tunstall, 1771), tree pipit (Anthus trivialis Linnaeus, 1758), African hoopoe (Upupa epops Linnaeus, 1758), and Caspian plover (Charadrius asiaticus Pallas, 1773) are Palearctic migrants. Globally vulnerable species such as black crowned crane (Balearica pavonina Linnaeus, 1758) were also recorded in the Alatish National Park (Table 1).

Order Passeriformes had the highest number of species (47), followed by order Accipitriformes (17 species) and orders Columbiformes, Coraciiformes, and Pelecaniformes with 11 species each. Order Passeriformes had also the highest number of families (23) followed by order Charadriiformes (5), and orders Pelecaniformes, Coraciiformes, Piciformes, and Bucerotiformes had three families each (Figure 2).

3.2. Species Diversity. The riverine woodland and the bamboo woodland habitats harboured the highest $\left(\mathrm{H}^{\prime}=3.53\right)$ and the lowest $\left(\mathrm{H}^{\prime}=2.81\right)$ avian species diversity, 
TABLE 1: List of avian species recorded during the wet and dry seasons and their distribution in different habitats within the park.

\begin{tabular}{|c|c|c|c|c|c|c|c|c|c|c|}
\hline \multirow[b]{2}{*}{ Order } & \multirow[b]{2}{*}{ Family } & \multirow[b]{2}{*}{$\begin{array}{l}\text { Common } \\
\text { name }\end{array}$} & \multirow[b]{2}{*}{ Scientific name } & \multicolumn{4}{|c|}{ Habitat type } & \multicolumn{3}{|c|}{ Seasons } \\
\hline & & & & $\begin{array}{c}\text { Riverine } \\
\text { woodland }\end{array}$ & $\begin{array}{l}\text { Wooded } \\
\text { grassland }\end{array}$ & Woodland & $\begin{array}{c}\text { Bamboo } \\
\text { woodland }\end{array}$ & Wet & Dry & Both \\
\hline Columbiformes & Columbidae & $\begin{array}{c}\text { African } \\
\text { collared dove }\end{array}$ & $\begin{array}{c}\text { Streptopelia } \\
\text { roseogrisea } \\
\text { Sundevall, } 1857\end{array}$ & & $\checkmark$ & & & & $\checkmark$ & \\
\hline Columbiformes & Columbidae & $\begin{array}{l}\text { African } \\
\text { mourning } \\
\text { dove }\end{array}$ & $\begin{array}{c}\text { Streptopelia } \\
\text { decipiens } \\
\text { Hartlaub and } \\
\text { Finsch, } 1870\end{array}$ & & & & $\checkmark$ & & & $\checkmark$ \\
\hline Columbiformes & Columbidae & $\begin{array}{l}\text { Black-billed } \\
\text { wood dove }\end{array}$ & $\begin{array}{c}\text { Turtur } \\
\text { abyssinicus } \\
\text { Sharpe, } 1902\end{array}$ & $\checkmark$ & & & & & $\checkmark$ & \\
\hline Columbiformes & Columbidae & $\begin{array}{l}\text { Blue-spotted } \\
\text { wood dove }\end{array}$ & $\begin{array}{c}\text { Turtur afer } \\
\text { Linnaeus, } 1766\end{array}$ & & & $\checkmark$ & & & & $\checkmark$ \\
\hline Columbiformes & Columbidae & $\begin{array}{l}\text { Bruce's green } \\
\text { pigeon }\end{array}$ & $\begin{array}{l}\text { Treron waalia } \\
\text { Meyer, } 1793\end{array}$ & $\checkmark$ & & & & & & $\checkmark$ \\
\hline Columbiformes & Columbidae & $\begin{array}{l}\text { Laughing } \\
\text { dove }\end{array}$ & $\begin{array}{c}\text { Streptopelia } \\
\text { senegalensis } \\
\text { Linnaeus, } 1766\end{array}$ & $\checkmark$ & & & & & & $\checkmark$ \\
\hline Columbiformes & Columbidae & $\begin{array}{l}\text { Namaqua } \\
\text { dove }\end{array}$ & $\begin{array}{l}\text { Oena capensis } \\
\text { Linnaeus, } 1766\end{array}$ & & $\checkmark$ & & & $\checkmark$ & & \\
\hline Columbiformes & Columbidae & $\begin{array}{l}\text { Red-eyed } \\
\text { dove }\end{array}$ & $\begin{array}{l}\text { Streptopelia } \\
\text { semitorquata } \\
\text { Rüppell, } 1837\end{array}$ & & $\checkmark$ & & & $\checkmark$ & & \\
\hline Columbiformes & Columbidae & $\begin{array}{l}\text { Ring-necked } \\
\text { dove }\end{array}$ & $\begin{array}{c}\text { Streptopelia } \\
\text { capicola } \\
\text { Sundevall, } 1857\end{array}$ & $\checkmark$ & & & & & & $\checkmark$ \\
\hline Columbiformes & Columbidae & $\begin{array}{l}\text { Speckled } \\
\text { pigeon }\end{array}$ & $\begin{array}{c}\text { Columba guinea } \\
\text { Linnaeus, } 1758\end{array}$ & & & & $\checkmark$ & & & $\checkmark$ \\
\hline Columbiformes & Columbidae & $\begin{array}{l}\text { White- } \\
\text { collared } \\
\text { pigeon }\end{array}$ & $\begin{array}{c}\text { Columba } \\
\text { albitorques } \\
\text { Rüppell, } 1837\end{array}$ & $\checkmark$ & & & & & $\checkmark$ & \\
\hline Coraciiformes & Coraciidae & $\begin{array}{l}\text { Abyssinian } \\
\text { roller }\end{array}$ & $\begin{array}{c}\text { Coracias } \\
\text { abyssinicus } \\
\text { Hermann, } 1783\end{array}$ & $\checkmark$ & & & & & & $\checkmark$ \\
\hline Coraciiformes & Meropidae & $\begin{array}{l}\text { Blue- } \\
\text { breasted bee- } \\
\text { eater }\end{array}$ & $\begin{array}{c}\text { Merops } \\
\text { variegatus } \\
\text { Vieillot, } 1817\end{array}$ & & $\checkmark$ & & & & $\checkmark$ & \\
\hline Coraciiformes & Meropidae & $\begin{array}{l}\text { Red-throated } \\
\text { bee-eater }\end{array}$ & $\begin{array}{c}\text { Merops bulocki } \\
\text { Vieillot,1817 }\end{array}$ & & & & $\checkmark$ & $\checkmark$ & & \\
\hline Coraciiformes & Meropidae & $\begin{array}{l}\text { throated bee- } \\
\text { eater }\end{array}$ & $\begin{array}{l}\text { Merops } \\
\text { bullockoides } \\
\text { Smith, } 1834\end{array}$ & & & & $\checkmark$ & & & $\checkmark$ \\
\hline Coraciiformes & Meropidae & $\begin{array}{l}\text { Little bee- } \\
\quad \text { eater }\end{array}$ & $\begin{array}{c}\text { Merops pusillus } \\
\text { Statius Müller, } \\
1776\end{array}$ & & & & $\checkmark$ & & & $\checkmark$ \\
\hline Coraciiformes & Alcedinidae & $\begin{array}{c}\text { Giant } \\
\text { kingfisher }\end{array}$ & $\begin{array}{c}\text { Megaceryle } \\
\text { maxima Pallas, } \\
1769\end{array}$ & & $\checkmark$ & & & $\checkmark$ & & \\
\hline Coraciiformes & Alcedinidae & $\begin{array}{l}\text { Malachite } \\
\text { kingfisher }\end{array}$ & $\begin{array}{l}\text { Alcedo cristata } \\
\text { Pallas, } 1764\end{array}$ & $\checkmark$ & & & & & $\checkmark$ & \\
\hline Coraciiformes & Alcedinidae & $\begin{array}{l}\text { Woodland } \\
\text { kingfisher }\end{array}$ & $\begin{array}{c}\text { Halcyon } \\
\text { senegalensis } \\
\text { Linnaeus, } 1766\end{array}$ & & & $\checkmark$ & & $\checkmark$ & & \\
\hline Coraciiformes & Alcedinidae & $\begin{array}{c}\text { Pied } \\
\text { kingfisher }\end{array}$ & $\begin{array}{c}\text { Ceryle rudis } \\
\text { Linnaeus, } 1758\end{array}$ & $\checkmark$ & & & & & & $\checkmark$ \\
\hline Coraciiformes & Alcedinidae & $\begin{array}{l}\text { Half-collared } \\
\text { kingfisher }\end{array}$ & $\begin{array}{c}\text { Alcedo } \\
\text { semitorquata } \\
\text { Swainson, } 1823\end{array}$ & $\checkmark$ & & & & & & $\checkmark$ \\
\hline
\end{tabular}


TABle 1: Continued.

\begin{tabular}{|c|c|c|c|c|c|c|c|c|c|c|}
\hline \multirow[b]{2}{*}{ Order } & \multirow[b]{2}{*}{ Family } & \multirow{2}{*}{$\begin{array}{l}\text { Common } \\
\text { name }\end{array}$} & \multirow[b]{2}{*}{ Scientific name } & \multicolumn{4}{|c|}{ Habitat type } & \multicolumn{3}{|c|}{ Seasons } \\
\hline & & & & $\begin{array}{c}\text { Riverine } \\
\text { woodland }\end{array}$ & $\begin{array}{l}\text { Wooded } \\
\text { grassland }\end{array}$ & Woodland & $\begin{array}{c}\text { Bamboo } \\
\text { woodland }\end{array}$ & Wet & Dry & Both \\
\hline Coraciiformes & Alcedinidae & $\begin{array}{l}\text { Grey-headed } \\
\text { kingfisher }\end{array}$ & $\begin{array}{c}\text { Halcyon } \\
\text { leucocephala } \\
\text { Müller, } 1776 \\
\text { Zosterops }\end{array}$ & $\checkmark$ & & & & & & $\checkmark$ \\
\hline Passeriformes & Zosteropidae & $\begin{array}{l}\text { Abyssinian } \\
\text { white eye }\end{array}$ & $\begin{array}{l}\text { abyssinicus } \\
\text { Guérin- } \\
\text { Méneville, } 1843\end{array}$ & & & $\checkmark$ & & $\checkmark$ & & \\
\hline Passeriformes & Fringillidae & African citril & $\begin{array}{c}\text { Crithagra } \\
\text { citrinelloides } \\
\text { Rüppell, } 1840\end{array}$ & $\checkmark$ & & & & & $\checkmark$ & \\
\hline Passeriformes & Alaudidae & $\begin{array}{l}\text { African } \\
\text { singing } \\
\text { bushlark }\end{array}$ & $\begin{array}{c}\text { Mirafra } \\
\text { cantillans } \\
\text { Blyth,1845 }\end{array}$ & & $\checkmark$ & & & & & $\checkmark$ \\
\hline Passeriformes & Turdidae & $\begin{array}{l}\text { African } \\
\text { thrush }\end{array}$ & $\begin{array}{c}\text { Turdus pelios } \\
\text { Bonaparte, } 1850\end{array}$ & & $\checkmark$ & & & & & $\checkmark$ \\
\hline Passeriformes & Turdidae & $\begin{array}{l}\text { Mountain } \\
\text { thrush }\end{array}$ & $\begin{array}{c}\text { Turdus } \\
\text { abyssinicus } \\
\text { Gmelin, } 1789\end{array}$ & & & $\checkmark$ & & & & $\checkmark$ \\
\hline Passeriformes & Muscicapidae & Familiar chat & $\begin{array}{c}\text { Oenanthe } \\
\text { familiaris } \\
\text { Wilkes, } 1817\end{array}$ & & & $\checkmark$ & & & & $\checkmark$ \\
\hline Passeriformes & Muscicapidae & $\begin{array}{c}\text { Brown-tailed } \\
\text { chat }\end{array}$ & $\begin{array}{c}\text { Oenanthe } \\
\text { scotocerca } \\
\text { Heuglin, } 1869\end{array}$ & & & & $\checkmark$ & & & $\checkmark$ \\
\hline Passeriformes & Viduidae & $\begin{array}{l}\text { Paradise } \\
\text { whydah }\end{array}$ & $\begin{array}{c}\text { Vidua } \\
\text { paradisaea } \\
\text { Linnaeus, } 1758\end{array}$ & & $\checkmark$ & & & $\checkmark$ & & \\
\hline Passeriformes & Viduidae & $\begin{array}{l}\text { Pin-tailed } \\
\text { whydah }\end{array}$ & $\begin{array}{c}\text { Vidua macroura } \\
\text { Pallas, } 1764\end{array}$ & & $\checkmark$ & & & $\checkmark$ & & \\
\hline Passeriformes & Viduidae & $\begin{array}{c}\text { Village } \\
\text { indigo bird }\end{array}$ & $\begin{array}{l}\text { Vidua chalybeate } \\
\text { Müller, } 1776\end{array}$ & & & & $\checkmark$ & & & $\checkmark$ \\
\hline Passeriformes & Estrildidae & $\begin{array}{l}\text { African } \\
\text { firefinch }\end{array}$ & $\begin{array}{c}\text { Lagonosticta } \\
\text { rubricata } \\
\text { Lichtenstein, } \\
1823\end{array}$ & & & & $\checkmark$ & $\checkmark$ & & \\
\hline Passeriformes & Estrildidae & $\begin{array}{l}\text { Cut-throat } \\
\text { finch }\end{array}$ & $\begin{array}{c}\text { Amadina fasciata } \\
\text { Gmelin, } 1789\end{array}$ & & & & $\checkmark$ & $\checkmark$ & & \\
\hline Passeriformes & Estrildidae & $\begin{array}{l}\text { Red-cheeked } \\
\text { cordon bleu }\end{array}$ & $\begin{array}{c}\text { Uraeginthus } \\
\text { bengalus } \\
\text { Linnaeus, } 1766\end{array}$ & & $\checkmark$ & & & & & $\checkmark$ \\
\hline Passeriformes & Estrildidae & $\begin{array}{l}\text { Bar breasted } \\
\text { firefinch }\end{array}$ & $\begin{array}{c}\text { Lagonosticta } \\
\text { rufopicta Fraster, } \\
1843\end{array}$ & & $\checkmark$ & & & $\checkmark$ & & \\
\hline Passeriformes & Nectariniidae & $\begin{array}{l}\text { Beautiful } \\
\text { sunbird }\end{array}$ & $\begin{array}{c}\text { Cinnyris } \\
\text { pulchellus } \\
\text { Linnaeus, } 1758\end{array}$ & & $\checkmark$ & & & $\checkmark$ & & \\
\hline Passeriformes & Malaconotidae & $\begin{array}{l}\text { Black-headed } \\
\text { gonolek }\end{array}$ & $\begin{array}{c}\text { Laniarius } \\
\text { erythrogaster } \\
\text { Cretzschmar, } \\
1829\end{array}$ & & & & $\checkmark$ & & & $\checkmark$ \\
\hline Passeriformes & Malaconotidae & $\begin{array}{l}\text { Black-headed } \\
\text { gonolek }\end{array}$ & $\begin{array}{c}\text { Laniarius } \\
\text { erythrogaster } \\
\text { Cretzschmar, } \\
1829\end{array}$ & & $\checkmark$ & & & & & $\checkmark$ \\
\hline Passeriformes & Leiothricidae & $\begin{array}{l}\text { White- } \\
\text { headed } \\
\text { babbler }\end{array}$ & $\begin{array}{c}\text { Turdoides } \\
\text { leucocephala } \\
\text { Cretzschmar, } \\
1827\end{array}$ & & & & $\checkmark$ & $\checkmark$ & & \\
\hline
\end{tabular}


TABle 1: Continued.

\begin{tabular}{|c|c|c|c|c|c|c|c|c|c|c|}
\hline \multirow[b]{2}{*}{ Order } & \multirow[b]{2}{*}{ Family } & \multirow{2}{*}{$\begin{array}{l}\text { Common } \\
\text { name }\end{array}$} & \multirow[b]{2}{*}{ Scientific name } & \multicolumn{4}{|c|}{ Habitat type } & \multicolumn{3}{|c|}{ Seasons } \\
\hline & & & & $\begin{array}{c}\text { Riverine } \\
\text { woodland }\end{array}$ & $\begin{array}{l}\text { Wooded } \\
\text { grassland }\end{array}$ & Woodland & $\begin{array}{c}\text { Bamboo } \\
\text { woodland }\end{array}$ & Wet & Dry & Both \\
\hline Passeriformes & Passeridae & $\begin{array}{c}\text { Bush } \\
\text { petronia }\end{array}$ & $\begin{array}{l}\text { Petronia dentata } \\
\text { Sundevall, } 1850\end{array}$ & & $\checkmark$ & & & & & $\checkmark$ \\
\hline Passeriformes & Ploceidae & $\begin{array}{l}\text { Village } \\
\text { weaver }\end{array}$ & $\begin{array}{c}\text { Ploceus } \\
\text { cucullatus } \\
\text { Müller, } 1776 \\
\text { Ploceus }\end{array}$ & & & & & & & \\
\hline Passeriformes & Ploceidae & Juba weaver & $\begin{array}{l}\text { dichrocephalus } \\
\text { Salvadori, } 1896\end{array}$ & $\checkmark$ & & & & $\checkmark$ & & \\
\hline Passeriformes & Ploceidae & $\begin{array}{c}\text { Black-headed } \\
\text { weaver }\end{array}$ & $\begin{array}{c}\text { Ploceus } \\
\text { melanocephalus } \\
\text { Linnaeus, } 1758\end{array}$ & & & $\checkmark$ & & & $\checkmark$ & \\
\hline Passeriformes & Ploceidae & $\begin{array}{l}\text { Chestnut } \\
\text { weaver }\end{array}$ & $\begin{array}{c}\text { Ploceus } \\
\text { rubiginosus } \\
\text { Rüppell, } 1840\end{array}$ & & & $\checkmark$ & & & $\checkmark$ & \\
\hline Passeriformes & Ploceidae & $\begin{array}{l}\text { Yellow- } \\
\text { crowned } \\
\text { bishop }\end{array}$ & $\begin{array}{l}\text { Euplectes afer } \\
\text { Gmelin, } 1789\end{array}$ & & $\checkmark$ & & & & $\checkmark$ & \\
\hline Passeriformes & Ploceidae & $\begin{array}{l}\text { Speke's } \\
\text { weaver }\end{array}$ & $\begin{array}{l}\text { Ploceus spekei } \\
\text { Heuglin, } 1861\end{array}$ & & $\checkmark$ & & & & $\checkmark$ & \\
\hline Passeriformes & Ploceidae & $\begin{array}{l}\text { Northern red } \\
\text { bishop }\end{array}$ & $\begin{array}{l}\text { Euplectes } \\
\text { franciscanus } \\
\text { Isert, } 1789\end{array}$ & & & $\checkmark$ & $\checkmark$ & & & \\
\hline Passeriformes & Ploceidae & $\begin{array}{l}\text { Rüppell's } \\
\text { weaver }\end{array}$ & $\begin{array}{l}\text { Ploceus galbula } \\
\text { Rüppell, } 1840 \\
\text { Ploceus luteolus }\end{array}$ & $\checkmark$ & & & & & $\checkmark$ & \\
\hline Passeriformes & Ploceidae & Little weaver & $\begin{array}{c}\text { Lichtenstein, } \\
1823\end{array}$ & & & $\checkmark$ & & $\checkmark$ & & \\
\hline Passeriformes & Ploceidae & $\begin{array}{l}\text { Black-winged } \\
\text { red bishop }\end{array}$ & $\begin{array}{c}\text { Euplectes } \\
\text { hordeaceus } \\
\text { Linnaeus, } 1758\end{array}$ & $\checkmark$ & & & $\checkmark$ & & & \\
\hline Passeriformes & Passeridae & $\begin{array}{l}\text { Northern } \\
\text { grey-headed } \\
\text { sparrow }\end{array}$ & $\begin{array}{l}\text { Passer griseus } \\
\text { Vieillot, } 1817\end{array}$ & & $\checkmark$ & & & $\checkmark$ & & \\
\hline Passeriformes & Cisticolidae & $\begin{array}{l}\text { Buff-belled } \\
\text { warbler }\end{array}$ & $\begin{array}{c}\text { Phyllolais } \\
\text { pulchella } \\
\text { Cretzschmar, } \\
1827\end{array}$ & & $\checkmark$ & & & & $\checkmark$ & \\
\hline Passeriformes & Pycnonotidae & $\begin{array}{l}\text { Common } \\
\text { bulbul }\end{array}$ & $\begin{array}{c}\text { Pycnonotus } \\
\text { barbatus } \\
\text { Desfontaines, } \\
1789\end{array}$ & & & $\checkmark$ & & $\checkmark$ & & \\
\hline Passeriformes & Corvidae & $\begin{array}{l}\text { Fan-tailed } \\
\text { raven }\end{array}$ & $\begin{array}{c}\text { Corvus } \\
\text { rhipidurus } \\
\text { Hartert, } 1918\end{array}$ & & $\checkmark$ & & & $\checkmark$ & & \\
\hline Passeriformes & Motacillidae & Golden pipit & $\begin{array}{c}\text { Timetothylacus } \\
\text { tenellus Cabanis, } \\
1878\end{array}$ & & & $\checkmark$ & & & & $\checkmark$ \\
\hline Passeriformes & Motacillidae & $\begin{array}{c}\text { Mountain } \\
\text { wagtail }\end{array}$ & $\begin{array}{l}\text { Motacilla clara } \\
\text { Sharpe, } 1908\end{array}$ & & & $\checkmark$ & & & & $\checkmark$ \\
\hline Passeriformes & Motacillidae & Tree pipit & $\begin{array}{l}\text { Anthus trivialis } \\
\text { Linnaeus, } 1758\end{array}$ & & & $\checkmark$ & & & $\checkmark$ & \\
\hline Passeriformes & Motacillidae & Pied wagtail & $\begin{array}{l}\text { Motacilla alba } \\
\text { Linnaeus, } 1758\end{array}$ & & $\checkmark$ & & & $\checkmark$ & & \\
\hline Passeriformes & Motacillidae & $\begin{array}{l}\text { Long-billed } \\
\text { pipit }\end{array}$ & $\begin{array}{l}\text { Anthus similis } \\
\text { Jerdon, } 1840\end{array}$ & & & $\checkmark$ & $\checkmark$ & & & \\
\hline Passeriformes & Motacillidae & $\begin{array}{l}\text { Plain-backed } \\
\text { pipit }\end{array}$ & $\begin{array}{c}\text { Anthus } \\
\text { leucophrys } \\
\text { Vieillot, } 1818\end{array}$ & & $\checkmark$ & & & $\checkmark$ & & \\
\hline
\end{tabular}


TABle 1: Continued.

\begin{tabular}{|c|c|c|c|c|c|c|c|c|c|c|}
\hline \multirow[b]{2}{*}{ Order } & \multirow[b]{2}{*}{ Family } & \multirow[b]{2}{*}{$\begin{array}{l}\text { Common } \\
\text { name }\end{array}$} & \multirow[b]{2}{*}{ Scientific name } & \multicolumn{4}{|c|}{ Habitat type } & \multicolumn{3}{|c|}{ Seasons } \\
\hline & & & & $\begin{array}{l}\text { Riverine } \\
\text { woodland }\end{array}$ & $\begin{array}{l}\text { Wooded } \\
\text { grassland }\end{array}$ & Woodland & $\begin{array}{l}\text { Bamboo } \\
\text { woodland }\end{array}$ & Wet & Dry & Both \\
\hline Passeriformes & Motacillidae & Grey wagtail & $\begin{array}{c}\text { Motacilla cinerea } \\
\text { Tunstall, } 1771\end{array}$ & & & $\checkmark$ & $\checkmark$ & & & \\
\hline Passeriformes & Locustellidae & $\begin{array}{l}\text { Little rush } \\
\text { warbler }\end{array}$ & $\begin{array}{c}\text { Bradypterus } \\
\text { baboecala } \\
\text { Vieillot, } 1817\end{array}$ & & $\checkmark$ & & & & $\checkmark$ & \\
\hline Passeriformes & Laniidae & $\begin{array}{l}\text { Nubian } \\
\text { shrike }\end{array}$ & $\begin{array}{c}\text { Lanius nubicus } \\
\text { Lichtenstein, } \\
1823\end{array}$ & & $\checkmark$ & & & & & $\checkmark$ \\
\hline Passeriformes & Acrocephalidae & $\begin{array}{l}\text { Eastern } \\
\text { olivaceous } \\
\text { warbler }\end{array}$ & $\begin{array}{c}\text { Iduna pallida } \\
\text { Hemprich and } \\
\text { Ehrenberg, } 1833\end{array}$ & & $\checkmark$ & & & & & $\checkmark$ \\
\hline Passeriformes & Cisticolidae & $\begin{array}{l}\text { Red-pate } \\
\text { cisticola }\end{array}$ & $\begin{array}{c}\text { Cisticola ruficeps } \\
\text { Cretzschmar, } \\
1827\end{array}$ & & $\checkmark$ & & $\checkmark$ & & & \\
\hline Passeriformes & Sturnidae & $\begin{array}{l}\text { Rüppel's } \\
\text { starling }\end{array}$ & $\begin{array}{l}\text { Lamprotornis } \\
\text { purpuroptera } \\
\text { Rüppell, } 1845\end{array}$ & & & $\checkmark$ & & & & $\checkmark$ \\
\hline Passeriformes & Dicruridae & $\begin{array}{l}\text { Square-tailed } \\
\text { drongo }\end{array}$ & $\begin{array}{l}\text { Dicrurus ludwigii } \\
\text { Smith, } 1834\end{array}$ & & & & $\checkmark$ & $\checkmark$ & & \\
\hline Passeriformes & Dicruridae & $\begin{array}{l}\text { Fork-tailed } \\
\text { drongo }\end{array}$ & $\begin{array}{c}\text { Dicrurus } \\
\text { adsimilis } \\
\text { Bechstein, } 1794\end{array}$ & & & & $\checkmark$ & $\checkmark$ & & \\
\hline Accipitriformes & Accipitridae & $\begin{array}{c}\text { African } \\
\text { hawk-eagle }\end{array}$ & $\begin{array}{c}\text { Aquila } \\
\text { spilogaster } \\
\text { Bonaparte, } 1850\end{array}$ & & & $\checkmark$ & & $\checkmark$ & & \\
\hline Accipitriformes & Accipitridae & $\begin{array}{l}\text { African fish- } \\
\text { eagle }\end{array}$ & $\begin{array}{c}\text { Haliaeetus } \\
\text { vocifer Daudin, } \\
1800\end{array}$ & & & $\checkmark$ & & $\checkmark$ & & \\
\hline Accipitriformes & Accipitridae & Black kite & $\begin{array}{l}\text { Milvus migrans } \\
\text { Boddaert, } 1783\end{array}$ & & & $\checkmark$ & $\checkmark$ & & & \\
\hline Accipitriformes & Accipitridae & Tawny eagle & $\begin{array}{c}\text { Aquila rapax } \\
\text { Temminck, } 1828\end{array}$ & $\checkmark$ & & & & & & $\checkmark$ \\
\hline Accipitriformes & Accipitridae & $\begin{array}{l}\text { Black-winged } \\
\text { kite }\end{array}$ & $\begin{array}{c}\text { Elanus caeruleus } \\
\text { Desfontaines, } \\
1789\end{array}$ & $\checkmark$ & & & & & & $\checkmark$ \\
\hline Accipitriformes & Accipitridae & $\begin{array}{c}\text { Brown snake } \\
\text { eagle }\end{array}$ & $\begin{array}{c}\text { Circaetus } \\
\text { cinereus Vieillot, } \\
1817\end{array}$ & & $\checkmark$ & & & & $\checkmark$ & \\
\hline Accipitriformes & Accipitridae & $\begin{array}{l}\text { Grasshopper } \\
\text { buzzard }\end{array}$ & $\begin{array}{l}\text { Butastur } \\
\text { rufipennis } \\
\text { Sundevall, } 1851\end{array}$ & & & $\checkmark$ & & $\checkmark$ & & \\
\hline Accipitriformes & Accipitridae & $\begin{array}{l}\text { Hooded } \\
\text { vulture }\end{array}$ & $\begin{array}{c}\text { Necrosyrtes } \\
\text { monachus } \\
\text { Temminck, } 1828\end{array}$ & & $\checkmark$ & & $\checkmark$ & & & \\
\hline Accipitriformes & Accipitridae & $\begin{array}{l}\text { Lappet-faced } \\
\text { vulture }\end{array}$ & $\begin{array}{c}\text { Torgos } \\
\text { tracheliotos } \\
\text { Foster, } 1791\end{array}$ & & $\checkmark$ & & & $\checkmark$ & & \\
\hline Accipitriformes & Accipitridae & $\begin{array}{l}\text { Long-crested } \\
\text { eagle }\end{array}$ & $\begin{array}{c}\text { Lophaetus } \\
\text { occipitalis } \\
\text { Daudin, } 1800\end{array}$ & & $\checkmark$ & & & $\checkmark$ & & \\
\hline Accipitriformes & Accipitridae & Martial eagle & $\begin{array}{c}\text { Polemaetus } \\
\text { bellicosus } \\
\text { Daudin, } 1800\end{array}$ & & $\checkmark$ & & & $\checkmark$ & & \\
\hline Accipitriformes & Accipitridae & Shikra & $\begin{array}{c}\text { Accipiter badius } \\
\text { Gmelin, } 1788\end{array}$ & & $\checkmark$ & & $\checkmark$ & & & \\
\hline Accipitriformes & Accipitridae & $\begin{array}{l}\text { Short-toed } \\
\text { snake eagle }\end{array}$ & $\begin{array}{c}\text { Circaetus gallicus } \\
\text { Gmelin, } 1788\end{array}$ & & $\checkmark$ & & & $\checkmark$ & & \\
\hline
\end{tabular}


TABle 1: Continued.

\begin{tabular}{|c|c|c|c|c|c|c|c|c|c|c|}
\hline \multirow[b]{2}{*}{ Order } & \multirow[b]{2}{*}{ Family } & \multirow{2}{*}{$\begin{array}{l}\text { Common } \\
\text { name }\end{array}$} & \multirow[b]{2}{*}{ Scientific name } & \multicolumn{4}{|c|}{ Habitat type } & \multicolumn{3}{|c|}{ Seasons } \\
\hline & & & & $\begin{array}{c}\text { Riverine } \\
\text { woodland }\end{array}$ & $\begin{array}{l}\text { Wooded } \\
\text { grassland }\end{array}$ & Woodland & $\begin{array}{c}\text { Bamboo } \\
\text { woodland }\end{array}$ & Wet & Dry & Both \\
\hline Accipitriformes & Accipitridae & $\begin{array}{l}\text { White- } \\
\text { headed } \\
\text { vulture }\end{array}$ & $\begin{array}{c}\text { Aegypius } \\
\text { occipitalis } \\
\text { Burchell, } 1824\end{array}$ & & $\checkmark$ & & & & $\checkmark$ & \\
\hline Accipitriformes & Accipitridae & $\begin{array}{l}\text { Lizard } \\
\text { buzzard }\end{array}$ & $\begin{array}{c}\text { Kaupifalco } \\
\text { monogrammicus } \\
\text { Temminck, } 1824\end{array}$ & & $\checkmark$ & & & $\checkmark$ & & \\
\hline Accipitriformes & Accipitridae & $\begin{array}{l}\text { Long-legged } \\
\text { buzzard }\end{array}$ & $\begin{array}{c}\text { Buteo rufinus } \\
\text { Cretzschmar, } \\
1829\end{array}$ & & & $\checkmark$ & $\checkmark$ & & & \\
\hline Accipitriformes & Sagittariidae & $\begin{array}{l}\text { Secretary } \\
\text { bird }\end{array}$ & $\begin{array}{l}\text { Sagittarius } \\
\text { serpentarius } \\
\text { Miller, } 1779\end{array}$ & & & $\checkmark$ & $\checkmark$ & & & \\
\hline Gruiformes & Heliornithidae & $\begin{array}{l}\text { African } \\
\text { finfoot }\end{array}$ & $\begin{array}{c}\text { Podica } \\
\text { senegalensis } \\
\text { Vieillot, } 1817\end{array}$ & & $\checkmark$ & & & $\checkmark$ & & \\
\hline Gruiformes & Gruidae & $\begin{array}{l}\text { Black- } \\
\text { crowned } \\
\text { crane }\end{array}$ & $\begin{array}{c}\text { Balearica } \\
\text { pavonina } \\
\text { Linnaeus, } 1758\end{array}$ & & $\checkmark$ & & & & $\checkmark$ & \\
\hline Suliformes & Phalacrocoracidae & $\begin{array}{l}\text { Long-tailed } \\
\text { cormorant }\end{array}$ & $\begin{array}{l}\text { Microcarbo } \\
\text { africanus } \\
\text { Gmelin, } 1789\end{array}$ & & & & $\checkmark$ & & & $\checkmark$ \\
\hline Pelecaniformes & Ardeidae & Cattle egret & $\begin{array}{c}\text { Bubulcus ibis } \\
\text { Linnaeus, } 1758\end{array}$ & & & $\checkmark$ & & & $\checkmark$ & \\
\hline Pelecaniformes & Ardeidae & $\begin{array}{l}\text { Yellow-billed } \\
\text { egret }\end{array}$ & $\begin{array}{c}\text { Egretta } \\
\text { intermedia } \\
\text { Wagler, } 1827\end{array}$ & & $\checkmark$ & & & $\checkmark$ & & \\
\hline Pelecaniformes & Ardeidae & $\begin{array}{l}\text { Black-headed } \\
\text { heron }\end{array}$ & $\begin{array}{c}\text { Ardea } \\
\text { melanocephala } \\
\text { Vigors, } 1826\end{array}$ & & & $\checkmark$ & & & $\checkmark$ & \\
\hline Pelecaniformes & Ardeidae & $\begin{array}{c}\text { Great white } \\
\text { egret }\end{array}$ & $\begin{array}{c}\text { Ardea alba } \\
\text { Linnaeus, } 1758\end{array}$ & & & $\checkmark$ & & & & $\checkmark$ \\
\hline Pelecaniformes & Ardeidae & $\begin{array}{l}\text { Common } \\
\text { squacco } \\
\text { heron }\end{array}$ & $\begin{array}{l}\text { Ardeola ralloides } \\
\text { Scopoli, } 1769\end{array}$ & & & $\checkmark$ & & $\checkmark$ & & \\
\hline Pelecaniformes & Ardeidae & Grey heron & $\begin{array}{l}\text { Ardea cinerea } \\
\text { Linnaeus, } 1758\end{array}$ & & & $\checkmark$ & $\checkmark$ & & & \\
\hline Pelecaniformes & Ardeidae & $\begin{array}{l}\text { Goliath } \\
\text { heron }\end{array}$ & $\begin{array}{c}\text { Ardea goliath } \\
\text { Cretzschmar, } \\
1827\end{array}$ & $\checkmark$ & & & & $\checkmark$ & & \\
\hline Pelecaniformes & Threskiornithidae & Glossy ibis & $\begin{array}{c}\text { Plegadis } \\
\text { falcinellus } \\
\text { Linnaeus, } 1766\end{array}$ & & $\checkmark$ & & & $\checkmark$ & & \\
\hline Pelecaniformes & Threskiornithidae & $\begin{array}{c}\text { African } \\
\text { sacred ibis }\end{array}$ & $\begin{array}{l}\text { Threskiornis } \\
\text { aethiopicus } \\
\text { Latham, } 1790\end{array}$ & & & $\checkmark$ & & & $\checkmark$ & \\
\hline Pelecaniformes & Threskiornithidae & Hadada ibis & $\begin{array}{c}\text { Bostrychia } \\
\text { hagedash } \\
\text { Latham, } 1790\end{array}$ & & $\checkmark$ & & & & $\checkmark$ & \\
\hline Pelecaniformes & Scopidae & Hammer kop & $\begin{array}{l}\text { Scopus umbretta } \\
\text { Gmelin, } 1789\end{array}$ & & & $\checkmark$ & & & $\checkmark$ & \\
\hline Ciconiiformes & Ciconiidae & White stork & $\begin{array}{l}\text { Ciconia ciconia } \\
\text { Linnaeus, } 1758\end{array}$ & & & & $\checkmark$ & & & $\checkmark$ \\
\hline Ciconiiformes & Ciconiidae & $\begin{array}{c}\text { Woolly } \\
\text { necked stork }\end{array}$ & $\begin{array}{c}\text { Ciconia episcopus } \\
\text { Boddaert, } 1783\end{array}$ & & & & $\checkmark$ & & $\checkmark$ & \\
\hline Ciconiiformes & Ciconiidae & Black stork & $\begin{array}{l}\text { Ciconia nigra } \\
\text { Linnaeus, } 1758\end{array}$ & $\checkmark$ & & & & & $\checkmark$ & \\
\hline
\end{tabular}


TABle 1: Continued.

\begin{tabular}{|c|c|c|c|c|c|c|c|c|c|c|}
\hline \multirow[b]{2}{*}{ Order } & \multirow[b]{2}{*}{ Family } & \multirow{2}{*}{$\begin{array}{l}\text { Common } \\
\text { name }\end{array}$} & \multirow[b]{2}{*}{ Scientific name } & \multicolumn{4}{|c|}{ Habitat type } & \multicolumn{3}{|c|}{ Seasons } \\
\hline & & & & $\begin{array}{c}\text { Riverine } \\
\text { woodland }\end{array}$ & $\begin{array}{l}\text { Wooded } \\
\text { grassland }\end{array}$ & Woodland & $\begin{array}{c}\text { Bamboo } \\
\text { woodland }\end{array}$ & Wet & Dry & Both \\
\hline Charadriiformes & Recurvirostridae & $\begin{array}{l}\text { Black-winged } \\
\text { stilt }\end{array}$ & $\begin{array}{c}\text { Himantopus } \\
\text { himantopus } \\
\text { Linnaeus, } 1758\end{array}$ & & & $\checkmark$ & & & $\checkmark$ & \\
\hline Charadriiformes & Charadriidae & $\begin{array}{l}\text { Caspian } \\
\text { plover }\end{array}$ & $\begin{array}{c}\text { Charadrius } \\
\text { asiaticus Pallas, } \\
1773\end{array}$ & & $\checkmark$ & & & $\checkmark$ & & \\
\hline Charadriiformes & Charadriidae & $\begin{array}{l}\text { Three- } \\
\text { banded } \\
\text { plover }\end{array}$ & $\begin{array}{c}\text { Charadrius } \\
\text { tricollaris } \\
\text { Vieillot, } 1818\end{array}$ & & $\checkmark$ & & & & $\checkmark$ & \\
\hline Charadriiformes & Charadriidae & $\begin{array}{l}\text { Kentish } \\
\text { plover }\end{array}$ & $\begin{array}{c}\text { Charadrius } \\
\text { alexandrinus } \\
\text { Linnaeus, } 1758\end{array}$ & $\checkmark$ & & & & & $\checkmark$ & \\
\hline Charadriiformes & Charadriidae & $\begin{array}{l}\text { Spur-winged } \\
\text { lapwing }\end{array}$ & $\begin{array}{l}\text { Vanellus spinosus } \\
\text { Linnaeus, } 1758\end{array}$ & $\checkmark$ & & & & & $\checkmark$ & \\
\hline Charadriiformes & Turnicidae & $\begin{array}{c}\text { Common } \\
\text { buttonquail }\end{array}$ & $\begin{array}{c}\text { Turnix sylvaticus } \\
\text { Desfontaines, } \\
1789\end{array}$ & $\checkmark$ & & & & & $\checkmark$ & \\
\hline Charadriiformes & Pluvianidae & $\begin{array}{l}\text { Egyptian } \\
\text { plover }\end{array}$ & $\begin{array}{c}\text { Pluvianus } \\
\text { aegyptius } \\
\text { Linnaeus, } 1758\end{array}$ & $\checkmark$ & & & & & $\checkmark$ & \\
\hline Charadriiformes & Burhinidae & $\begin{array}{c}\text { Senegal } \\
\text { thick-knee }\end{array}$ & $\begin{array}{c}\text { Burhinus } \\
\text { senegalensis } \\
\text { Swainson, } 1837\end{array}$ & $\checkmark$ & & & & $\checkmark$ & & \\
\hline Charadriiformes & Burhinidae & $\begin{array}{c}\text { Eurasian } \\
\text { stone curlew }\end{array}$ & $\begin{array}{c}\text { Burhinus } \\
\text { oedicnemus } \\
\text { Linnaeus, } 1758\end{array}$ & $\checkmark$ & & & & & & $\checkmark$ \\
\hline Charadriiformes & Burhinidae & $\begin{array}{l}\text { Water thick- } \\
\text { knee }\end{array}$ & $\begin{array}{c}\text { Burhinus } \\
\text { vermiculatus } \\
\text { Cabanis, } 1868\end{array}$ & & $\checkmark$ & & & $\checkmark$ & & \\
\hline Piciformes & Lybiidae & $\begin{array}{c}\text { Black-billed } \\
\text { barbet }\end{array}$ & $\begin{array}{c}\text { Lybius } \\
\text { guifsobalito } \\
\text { Hermann, } 1783\end{array}$ & & $\checkmark$ & & & & & $\checkmark$ \\
\hline Piciformes & Indicatoridae & $\begin{array}{c}\text { Lesser } \\
\text { honeyguide }\end{array}$ & $\begin{array}{l}\text { Indicator minor } \\
\text { Stephens, } 1815\end{array}$ & & & $\checkmark$ & & & & $\checkmark$ \\
\hline Piciformes & Picidae & $\begin{array}{c}\text { Nubian } \\
\text { woodpecker }\end{array}$ & $\begin{array}{c}\text { Campethera } \\
\text { nubica Boddaert, } \\
1783\end{array}$ & & $\checkmark$ & & & & $\checkmark$ & \\
\hline Galliformes & Phasianidae & $\begin{array}{l}\text { Clapperton’s } \\
\text { Francolin }\end{array}$ & $\begin{array}{c}\text { Pternistis } \\
\text { clappertoni } \\
\text { Vigors, } 1826\end{array}$ & $\checkmark$ & & & & $\checkmark$ & & \\
\hline Galliformes & Phasianidae & $\begin{array}{c}\text { Sand } \\
\text { partridge }\end{array}$ & $\begin{array}{c}\text { Ammoperdix } \\
\text { heyi Temminck, } \\
1825\end{array}$ & & $\checkmark$ & & & & & $\checkmark$ \\
\hline Galliformes & Phasianidae & $\begin{array}{c}\text { Scaly } \\
\text { Francolin }\end{array}$ & $\begin{array}{c}\text { Pternistis } \\
\text { squamatus } \\
\text { Cassin, } 1857\end{array}$ & & & $\checkmark$ & & & $\checkmark$ & \\
\hline Galliformes & Numididae & $\begin{array}{l}\text { Helmeted } \\
\text { guineafowl }\end{array}$ & $\begin{array}{c}\text { Numida } \\
\text { meleagris } \\
\text { Linnaeus, } 1758\end{array}$ & & & $\checkmark$ & & & $\checkmark$ & \\
\hline Strigiformes & Strigidae & $\begin{array}{l}\text { Common } \\
\text { scops owl }\end{array}$ & $\begin{array}{c}\text { Otus senegalensis } \\
\text { Swainson, } 1837\end{array}$ & & & $\checkmark$ & & $\checkmark$ & & \\
\hline Anseriformes & Anatidae & $\begin{array}{l}\text { Egyptian } \\
\text { goose }\end{array}$ & $\begin{array}{c}\text { Alopochen } \\
\text { aegyptiaca } \\
\text { Linnaeus, } 1766\end{array}$ & & & $\checkmark$ & & & $\checkmark$ & \\
\hline Bucerotiformes & Upupidae & $\begin{array}{l}\text { African } \\
\text { hoopoe }\end{array}$ & $\begin{array}{l}\text { Upupa africana } \\
\text { Bechstein,1811 }\end{array}$ & & $\checkmark$ & & & & $\checkmark$ & \\
\hline
\end{tabular}


TABle 1: Continued.

\begin{tabular}{|c|c|c|c|c|c|c|c|c|c|c|}
\hline \multirow[b]{2}{*}{ Order } & \multirow[b]{2}{*}{ Family } & \multirow[b]{2}{*}{$\begin{array}{l}\text { Common } \\
\text { name }\end{array}$} & \multirow[b]{2}{*}{ Scientific name } & \multicolumn{4}{|c|}{ Habitat type } & \multicolumn{3}{|c|}{ Seasons } \\
\hline & & & & $\begin{array}{c}\text { Riverine } \\
\text { woodland }\end{array}$ & $\begin{array}{l}\text { Wooded } \\
\text { grassland }\end{array}$ & Woodland & $\begin{array}{c}\text { Bamboo } \\
\text { woodland }\end{array}$ & Wet & Dry & Both \\
\hline Bucerotiformes & Phoeniculidae & $\begin{array}{l}\text { Green wood } \\
\text { hoopoe }\end{array}$ & $\begin{array}{c}\text { Phoeniculus } \\
\text { purpureus Miller, } \\
1784\end{array}$ & & $\checkmark$ & & & & $\checkmark$ & \\
\hline Bucerotiformes & Phoeniculidae & $\begin{array}{l}\text { Violet wood } \\
\text { hoopoe }\end{array}$ & $\begin{array}{c}\text { Phoeniculus } \\
\text { damarensis } \\
\text { Ogilvie-Grant, } \\
1901\end{array}$ & & & $\checkmark$ & & & & $\checkmark$ \\
\hline Bucerotiformes & Bucerotidae & $\begin{array}{l}\text { Northern } \\
\text { red-billed } \\
\text { hornbill }\end{array}$ & $\begin{array}{c}\text { Tockus } \\
\text { erythrorhynchus } \\
\text { Temminck, } 1823\end{array}$ & & $\checkmark$ & & & & & $\checkmark$ \\
\hline Cuculiformes & Cuculidae & $\begin{array}{l}\text { Levaillant's } \\
\text { cuckoo }\end{array}$ & $\begin{array}{c}\text { Clamator } \\
\text { levaillantii } \\
\text { Swainson, } 1829\end{array}$ & & & $\checkmark$ & & $\checkmark$ & & \\
\hline Cuculiformes & Cuculidae & $\begin{array}{l}\text { Senegal } \\
\text { coucal }\end{array}$ & $\begin{array}{c}\text { Centropus } \\
\text { senegalensis } \\
\text { Linnaeus, } 1766\end{array}$ & & & $\checkmark$ & & & $\checkmark$ & \\
\hline Cuculiformes & Cuculidae & $\begin{array}{l}\text { Blue-headed } \\
\text { coucal }\end{array}$ & $\begin{array}{c}\text { Centropus } \\
\text { monachus } \\
\text { Rüppell, } 1837\end{array}$ & & & & $\checkmark$ & & $\checkmark$ & \\
\hline Musophagiformes & Musophagidae & $\begin{array}{l}\text { White-belled } \\
\text { go-away-bird }\end{array}$ & $\begin{array}{c}\text { Crinifer } \\
\text { leucogaster } \\
\text { Rüppell, } 1842\end{array}$ & & & & $\checkmark$ & & & $\checkmark$ \\
\hline Psittaciformes & Psittaculidae & $\begin{array}{l}\text { Rose-ringed } \\
\text { parakeet }\end{array}$ & $\begin{array}{c}\text { Psittacula } \\
\text { krameri Scopoli, } \\
1769\end{array}$ & & & & $\checkmark$ & & & $\checkmark$ \\
\hline Coliformes & Coliidae & $\begin{array}{l}\text { Speckled } \\
\text { mousebird }\end{array}$ & $\begin{array}{l}\text { Colius striatus } \\
\text { Gmelin, } 1789\end{array}$ & $\checkmark$ & & & & & & $\checkmark$ \\
\hline
\end{tabular}

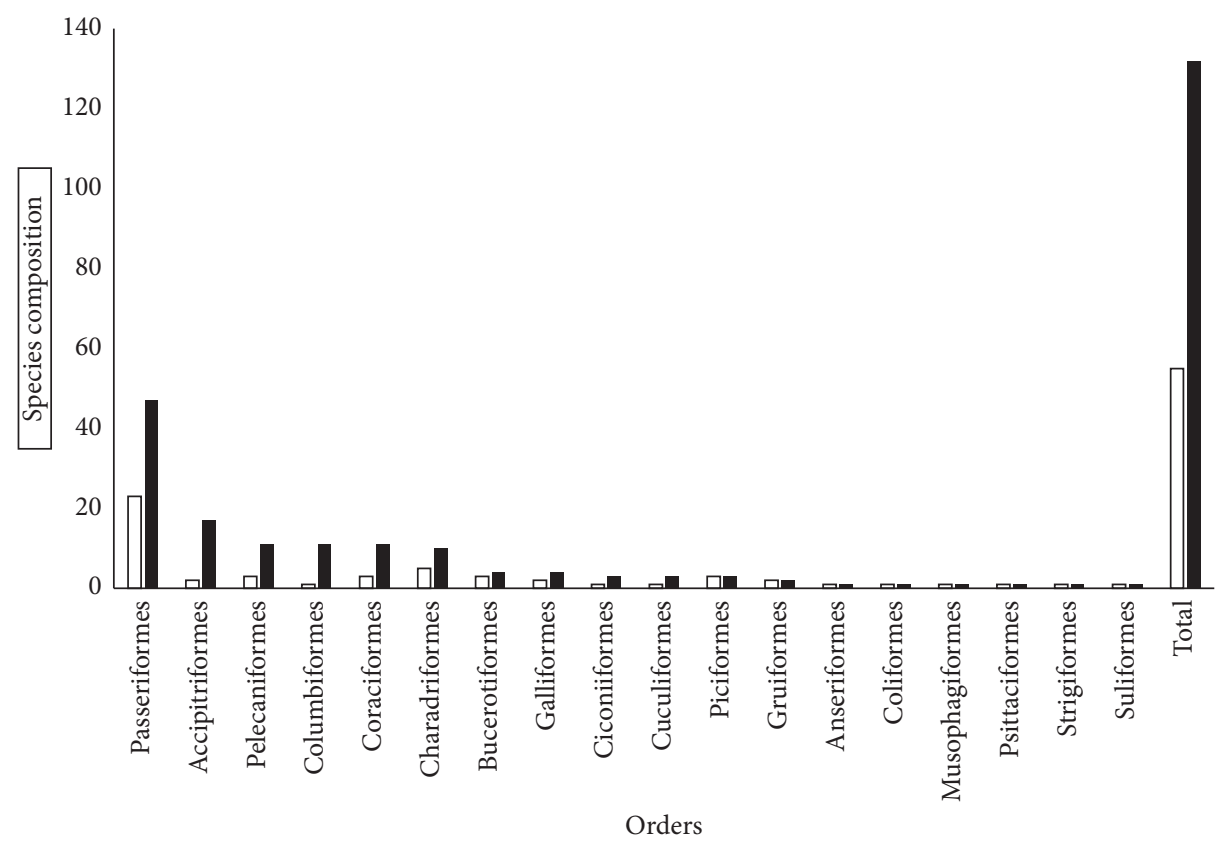

口 Number of Family

- Number of Species

Figure 2: Avian species composition in Alatish National Park. 
respectively, during the wet season. The species richness and abundance were the highest in the riverine woodland habitat, and the lowest was in the bamboo woodland habitat during the wet season. Species evenness was also the highest in the riverine woodland habitat during the wet season (Table 2).

During the dry season, the highest avian species diversity was recorded in the riverine woodland habitat $\left(\mathrm{H}^{\prime}=3.55\right)$, while the lowest was in the woodland habitat $\left(\mathrm{H}^{\prime}=2.45\right)$. A riverine woodland habitat harboured the highest avian species richness and abundance, while the lowest was in the woodland habitat during the dry season. During the same dry season, species evenness was also the highest in the riverine woodland habitat (Table 3).

During the whole study period, the highest species diversity and evenness were recorded in the riverine woodland habitat, and the lowest were in wooded grassland habitat (Table 4).

3.3. Species Similarity. Wet season avian species similarity result indicated that relatively the highest species similarity was recorded between the riverine woodland and wooded grassland habitats, while the lowest was between woodland and bamboo woodland habitats (Table 5).

During the dry season, relatively the highest avian species similarity occurred between wooded grassland and bamboo woodland habitats, and the lowest was between riverine woodland and wooded grassland habitats and between riverine woodland and woodland habitats (Table 6).

3.4. Relative Abundance. There was an association between relative abundance of birds and seasons. During the wet and dry seasons, individuals of 1,571 and 1,263 birds, respectively, were recorded in the park. There was a significant difference in abundance of birds among the difference species $\left(F_{131}, 1452=2.94, P<0.05\right)$. The most abundant bird species in the study area was Rüppell's weaver ( $P$. galbula), while the least abundance was for yellow-billed egret (A. brachyrhyncha).

During the wet season, the riverine woodland habitat had relatively the highest number of birds and the lowest was found in bamboo woodland habitat. During the dry season, the riverine woodland habitat had also relatively the highest number of birds, while the lowest was found from woodland habitat. According to Tukey's HSD test at $P=0.05$, means followed by the same letter(s) are not significantly different (Table 7).

Relative abundance of birds in Alatish National Park during the wet season showed that $125(47.2 \%)$ of the species were uncommon, 69 (26\%) frequent, $32(12 \%)$ common, and 39 (14.7\%) abundant. During the dry season, $108(44.0 \%)$ of bird species were uncommon, 72 (29.4\%) frequent, 43 (17.5\%) common, and 22 (9.0\%) abundant (Table 8).

3.5. Habitat Association. The highest habitat association of birds during the wet season was recorded in the riverine woodland habitat followed by wooded grassland habitat, while the lowest was in the bamboo woodland habitat. During the dry season, the highest habitat association of birds was also recorded in the riverine woodland habitat, while the lowest was in the woodland habitat (Figure 3).

\section{Discussion}

Most of the birds in Alatish National Park are uncommon species. This result is similar to $[33,34]$ indicating that the park is home to different groups of birds. Species diversity is an indication of habitat heterogeneity, and the numbers of species and individuals in an area imply the importance of the area in conservation [35]. During the wet season, most of the birds are distributed throughout the park since anthropogenic disturbance such as fire is not the problem in the park. However, during the dry season, burning of the natural vegetation affects avian breeding sites and food; as a result, they leave the area and move to remote habitats where human disturbance is relatively less common. Scarcity of water and food is also an important factor for birds to change their previous habitat ranges during the dry season. As a result, less species of birds were recorded during the dry season than during the wet season.

Some species occurring in the Alatish River during the wet season were found in the Hyma River during the dry season. This is attributed to the availability of adequate food and water in Hyma River habitats as the Alatish River dried up during the dry season. As a result, birds locally migrate from Alatish River to Hyma River within the park. There are also some species of birds that are observed in Alatish National Park only during the dry season. This might be associated with migration of birds where species from other continents such as Europe can travel to Africa to escape the bad weather during winter.

Higher plant composition in riverine woodland habitat of the park supports the maximum avian species. This is because species richness and abundance are influenced by local resource availability and vegetation composition in the given habitat. As a result, species have a nonuniform distribution in space, and this is also in line with the findings of other researchers [36]. Since the occurrence of different tree species provides different opportunities for foraging and nesting, the riverine woodland habitat is suitable for different avian species. Moreover, the occurrence of different microhabitats in the riverine woodland habitat favours birds to use for nesting and sheltering which are safe to prevent themselves from predators.

The riverine woodland habitat has the highest bird species diversity, richness, and evenness during the dry season, and this result is also in line with $[26,33]$. This is due to the occurrence of rich habitat diversity with both structure and species composition of plants in this habitat. Different tree species provide different opportunities for foraging and providing microhabitats. Similarly, the availability of water and cover during the dry season in the riverine woodland habitat contributes to the occurrence of maximum avian species diversity. Thus, availability of water and food is the main ecological requirement to determine diversity and relative abundance of avian species in Alatish National Park. As the number of vegetation layer increases, 
TABle 2: Avian diversity during the wet season.

\begin{tabular}{|c|c|c|c|c|c|c|}
\hline Habitat types & Species richness & Abundance & $\mathrm{H}^{\prime}$ & $\mathrm{H}^{\prime} \max$ & H'/Hax & $\mathrm{D}$ \\
\hline Riverine woodland & 89 & 5574 & 3.53 & 4.49 & 0.79 & 0.99 \\
\hline Wooded grassland & 80 & 4600 & 3.30 & 4.38 & 0.75 & 0.96 \\
\hline Woodland & 55 & 3011 & 3.03 & 4.00 & 0.75 & 0.95 \\
\hline Bamboo woodland & 41 & 2566 & 2.81 & 3.71 & 0.76 & 0.93 \\
\hline
\end{tabular}

Table 3: Avian diversity during the dry season.

\begin{tabular}{|c|c|c|c|c|c|c|}
\hline Habitat types & Species richness & Abundance & $\mathrm{H}^{\prime}$ & $\mathrm{H}^{\prime} \max$ & H'/Hax & $\mathrm{D}$ \\
\hline Riverine woodland & 110 & 6895 & 3.55 & 4.70 & 0.77 & 0.97 \\
\hline Wooded grassland & 56 & 1959 & 2.70 & 4.02 & 0.67 & 0.93 \\
\hline Woodland & 39 & 1484 & 2.45 & 3.66 & 0.67 & 0.91 \\
\hline Bamboo woodland & 40 & 2025 & 2.74 & 3.69 & 0.74 & 0.93 \\
\hline
\end{tabular}

TABLE 4: Avian diversity during both the wet and dry seasons.

\begin{tabular}{|c|c|c|c|c|c|c|}
\hline Habitat types & Species richness & Abundance & $\mathrm{H}^{\prime}$ & $\mathrm{H}^{\prime} \max$ & H'/Hax & $\mathrm{D}$ \\
\hline Riverine woodland & 129 & 8516 & 3.14 & 4.85 & 0.64 & 0.96 \\
\hline Wooded grassland & 57 & 2936 & 2.34 & 4.04 & 0.58 & 0.96 \\
\hline Woodland & 59 & 2398 & 2.43 & 4.07 & 0.59 & 0.87 \\
\hline Bamboo woodland & 53 & 3039 & 2.39 & 3.97 & 0.60 & 0.90 \\
\hline
\end{tabular}

TABLE 5: Avian species similarity among the four habitat types during the wet season.

\begin{tabular}{lccc}
\hline Habitat types & Wooded grassland & Woodland & Bamboo woodland \\
\hline Riverine woodland & 0.57 & 0.54 & 0.47 \\
Wooded grassland & & 0.50 & 0.46 \\
Woodland & & & 0.45 \\
Bamboo woodland & & & \\
\hline
\end{tabular}

TAвLE 6: Avian species similarity among the four habitat types during the dry season.

\begin{tabular}{lccc}
\hline Habitat types & Wooded grassland & Woodland & Bamboo woodland \\
\hline Riverine woodland & 0.67 & 0.67 & 0.70 \\
Wooded grassland & & 0.89 & 0.90 \\
Woodland & & & 0.75 \\
\hline
\end{tabular}

TABLE 7: Average number of birds counted at different habitats versus seasons.

\begin{tabular}{lccc}
\hline Habitat types & \multicolumn{2}{c}{ Seasons } \\
& Wet & Dry & Both wet and dry \\
\hline Riverine woodland & $43.3 \mathrm{a}$ & $39.8 \mathrm{AB}$ & $43.2 \mathrm{a}$ \\
Wooded grassland & $38.0 \mathrm{AB}$ & $14.2 \mathrm{DE}$ & $38.3 \mathrm{AB}$ \\
Woodland & $22.2 \mathrm{CDE}$ & $10.1 \mathrm{E}$ & $13.3 \mathrm{DE}$ \\
Bamboo woodland & $14.5 \mathrm{DE}$ & $31.1 \mathrm{ABC}$ & $25.4 \mathrm{BCD}$ \\
\hline
\end{tabular}

TABLE 8: Relative abundance of bird species during the wet and dry seasons.

\begin{tabular}{|c|c|c|c|c|c|}
\hline \multirow{2}{*}{ Habitat types } & \multicolumn{5}{|c|}{ Abundance rank } \\
\hline & Seasons & Uncommon & Frequent & Common & Abundant \\
\hline \multirow{2}{*}{ Riverine woodland } & Wet & 37 & 24 & 9 & 19 \\
\hline & dry & 40 & 36 & 21 & 13 \\
\hline \multirow{2}{*}{ Wooded grassland } & Wet & 31 & 21 & 17 & 11 \\
\hline & dry & 22 & 21 & 7 & 6 \\
\hline \multirow{2}{*}{ Woodland } & Wet & 34 & 11 & 4 & 6 \\
\hline & dry & 20 & 5 & 11 & 3 \\
\hline \multirow{2}{*}{ Bamboo woodland } & Wet & 23 & 13 & 2 & 3 \\
\hline & dry & 26 & 10 & 4 & - \\
\hline
\end{tabular}




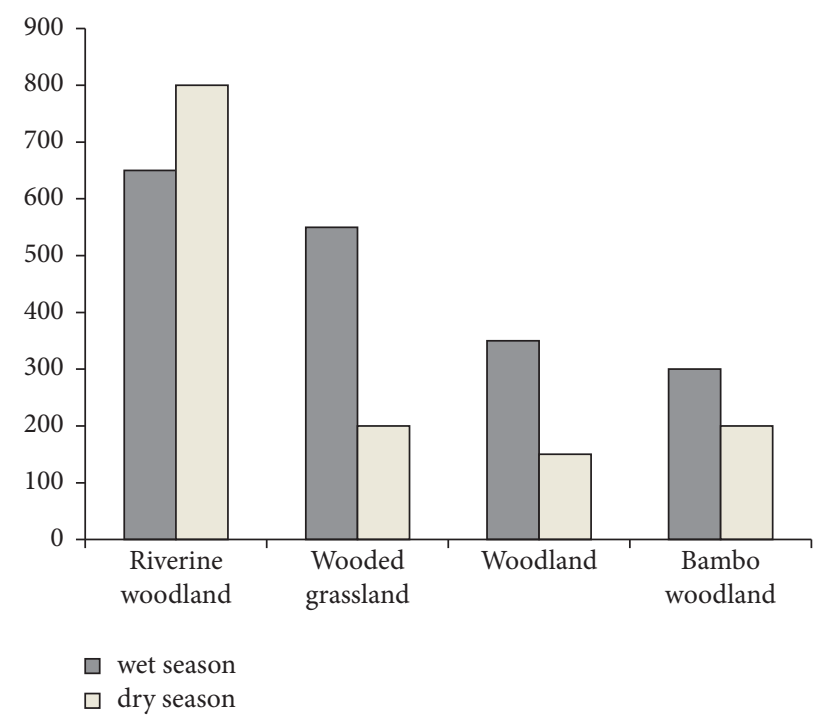

Figure 3: Avian species habitat association among the four habitat types.

the number of available niches for species also increases, and so does the diversity of the species $[13,37]$.

Small-sized birds, which belong to the order Passeriformes, are dominantly observed feeding, resting, and roosting on the grasses and bushy habitats of the park. Birds' response to wooded grassland habitats of different heights differed by species diversity due to the different patterns of habitat use. The availability of seed-bearing plants within the grasslands and the occurrence of different invertebrate species in grassland and bushy habitats can attract smallsized birds. In bamboo woodland habitat, the diversity and richness of birds during the wet season are relatively low. This might be because bamboo woodland habitats are sparsely distributed in the landscape, with limited available resources. Every habitat has a specific set of microenvironments which are suitable for species $[15,38]$. Therefore, habitat requirement of each species could determine its distribution in the park.

As anthropogenic disturbance such as forest fire in the park is not a common problem during the wet season, most of avian species are distributed in different habitat types within the park. However, during the dry season, frequent incidence of forest fire by the nomads and the local people in the park's natural habitat could affect the breeding ground, food, and cover of birds. Moreover, scarcity of water and food during the dry season could also lead birds to change their previous habitat ranges and become restricted to habitats where their ecological requirements are easily available. Avian species such as Fork-tailed drongo (D. adsimilis), Green wood hoopoe (P. purpureus), little beeeater (M. pusillus), and red-cheeked cordon bleu ( $U$. bengalus) are more common avian species along the Hyma River during the dry season. During the wet season, flowering plants are flourishing within the park and, as a result, food is plentiful for birds in almost all of the habitats. However, during the dry season, the deciduous trees have defoliated and, in the absence of food, many species of birds were restricted to riverine woodland habitat.
The differences in species composition and abundance among the four habitat types could be described as a function of the role that vegetation structure and composition in a given habitat can play in shaping the structure and organization of avian communities, and this is also in line with the findings of other researchers [39, 40]. The availability of different substrates for breeding, hiding, and foraging and different food items might attract different niche requiring bird species which result into higher species richness.

The wet season avian diversity result indicates relatively more species similarity between the riverine woodland and wooded grassland habitats because these habitats are similar in vegetation structure and composition. Aa a result, bird habitat association in these two habitats is nearly the same. In contrast, the low avian species similarity is recorded between woodland and bamboo woodland habitats. This is probably due to the difference in feeding adaptation of avian species in these slightly different habitats. Canopy closure supports more of habitat specialist avian species, while areas under anthropogenic influence harbour more of opportunistic species [41]. Moreover, similarity of avian species composition between habitats indicates a tendency for similar habitats to have similar species composition $[13,26,42]$.

The relative abundance of birds in the study area is correlated to the availability of food, habitat condition, and breeding season of the species. The distinct seasonality of the rainfall and seasonal variation in the abundance of food resource result in seasonal changes in abundance of birds. The number of uncommon species recorded in the bamboo woodland habitat is relatively high. These species which have low number of individuals relative to the effort made during the survey compared to others had grouped them in the uncommon category. This result can be associated with the degradation of bamboo woodland habitat which is important for feeding, resting, and breeding. In the present study area, habitat degradation is due to vegetation clearing, 
burning, and habitat modification. Such type of habitat disturbance and degradation would reduce the individual population of the species in many ways. The presence of a large number of uncommon species in a certain area could be related to the breeding nature, large home range, and niche of the species [43]. Moreover, the number of individual birds varied independently within the area. A few species abandon the specific area, decreasing in the numbers of both species and individuals, while others may have a smaller number of avian species that may present as a few spread individuals throughout the year.

In Alatish National Park, association of birds with a certain type of plant species, flower bearing trees, bushes, and grasses has been observed during the study period. The distribution and abundance of many avian species are determined by the composition of the vegetation that comprises a major element of their habitats [44]. Some bird species were more assembled with a particular plant species including fruiting trees, flowering trees, and bushes [45].

On the shore of the rivers where Diospyros mespiliformis, Anogeissus leiocarpus, and Ficus glumosa tress are dominant, African fish-eagle ( $H$. vocifer) is assembled to these tree species and such trees are used as its resting sites. As this avian species depends on fish, it needs such trees for resting and watching to dive in the water and capture its prey. Avian species diversity of Alatish National Park relates to the occurrence of different types of niches that exist within the park. Some of the habitats contain diverse niches, which increases the diversity and abundance of avian species, since some groups of birds feed on different invertebrates, some on flowers, some on seeds, and some on parts of the plants like leaves. The distribution, richness, and abundance of many avian species are determined by the pattern and composition of the habitat components comprising a major element of their habitat [46].

\section{Conclusion}

A total of 132 species of birds were recorded in Alatish National Park indicating that the park is rich in its avian diversity. The majority of avian species belong to the order Passeriformes followed by order Falconiformes. Order Passeriformes had also the highest number of families. Families Columbidae, Phasianidae Cuculidae, Accipitridae, Meropidae, Muscicapidae, Numididae, Phoeniculidae, and Dicruridae are found to be the most widely distributed families in the park.

Two near-endemic species were shared by Ethiopia and Eritrea, and six species which are African migrants, four Palearctic migrants, and one globally threatened species have been recorded in the park. The distribution of birds in different habitats is mainly determined due to the availability of food and water [13,47]. Accordingly, the riverine woodland habitat has the highest avian species diversity, evenness, and richness, and it is followed by wooded grassland habitat. The highest species diversity, evenness, and richness in the riverine woodland habitat revealed the importance of this habitat for biodiversity conservation.
Most of the avian species in Alatish National Park are locally uncommon and frequent, and the occurrence of threatened, endemic, uncommon, and vulnerable avian species makes the park an area of conservation importance. This revealed the need to give priority for conservation of birds in particular and the whole biological diversity in general. As a result, different anthropogenic activities including deliberate forest fire, logging, and overgrazing by nomadic cattle should be controlled or at least minimized.

\section{Data Availability}

All data used for this study are available from the corresponding author upon request.

\section{Conflicts of Interest}

The authors declare no conflicts of interest regarding the publication of this paper.

\section{Authors' Contributions}

TT has organized the data and feed to software for analysis. TT and DE proposed the research idea and participated in data collection in the park, carried out analysis, interpretation, and manuscript preparation, and read and approved the final manuscript.

\section{Acknowledgments}

The authors would like to thank field assistants and scouts of Alatish National Park for their prominent help during data collection time. The logistics support and hospitality given by the local people living around the park are highly acknowledged. The authors also thank the National Meteorological Service Agency, Bahir Dar Branch, for the provision of meteorological data. Trade, Industry and Market Development Bureau of Amhara National Regional State is acknowledged for the research fund provided to the first author. The corresponding author is very grateful to Professor Larkin Powell at University of Nebraska-Lincoln for arranging office facilities during this manuscript preparation.

\section{References}

[1] D. Lepage, "Avibase-Bird checklists of the World-Ethiopia," 2021, http://www.africanbirdclub.org.

[2] M. Wondafrash, "Wetlands, birds, and important bird areas in Ethiopia," in Wetlands of Ethiopia: Proceedings of a Seminar on the Resource and Status of 110 Ethiopia's Wetlands, Y. Delelegn and K. Geheb, Eds., pp. 25-30, International Union for Conservation of Nature and Natural Resources, Gland, Switzerland, 2003.

[3] S. Aynalem and A. Bekele, "Species composition, relative abundance, and distribution of bird fauna of riverine and wetland habitats of Infranz and Yiganda at southern tip of Lake Tana, Ethiopia," Tropical Ecology, vol. 49, no. 2, pp. 199-209, 2008.

[4] Handbook of the Birds of the World and BirdLife International Digital Checklist of the Birds of the World. Version 3, 
2018, http://datazone.birdlife.org/userfiles/file/Species/Taxonomy/HBWBirdLifeChecklistv3Nov18.zip.

[5] Bird Life International, Threatened birds of the World, CDROM, Bird Life International, Cambridge, UK, 2008, https:// www.birdlife.org/datazone/sowb/casestudy/114.

[6] J. A. Veech and T. O. Crist, "Habitat and climate heterogeneity maintain beta-diversity of birds among landscapes within ecoregions," Global Ecology and Biogeography, vol. 16, no. 5, pp. 650-656, 2007.

[7] C. J. Whelan, D. G. Wenny, and R. J. Marquis, "Ecosystem services provided by birds," Annals of the New York Academy of Sciences, vol. 1134, no. 1, pp. 25-60, 2008.

[8] W. G. Ware, "Complete guide to pest control: with and without chemicals. Thomson Publications," California, vol. 304, 1988.

[9] R. D. Gregory, D. Noble, J. H. Field, M. Marchant, and D. W. Gibbons, "Using birds as indicators of biodiversity," Ornis Hungarica, vol. 12, pp. 11-24, 2003.

[10] R. Rodríguez-Estrella, "Land use changes affect distributional patterns of desert birds in the Baja California Peninsula, Mexico," Diversity and Distributions, vol. 13, no. 6, pp. 877-889, 2007.

[11] V. Remes, "Effects of exotic habitat on nesting success, territory density, and settlement patterns in the blackcap (Sylvia atricapilla)," Conservation Biology, vol. 17, pp. 1127-1133, 2003.

[12] D. R. Petit, D. G. Petit, and H. D. W. Powell, "Bird communities of natural and modified habitats in Panama," Ecography, vol. 22, pp. 292-304, 1999.

[13] Y. Genet and D. Ejigu, "Community composition, relative abundance and habitat association of avian species in Apini and Dikuma forest patches, Awi Administrative Zone, Ethiopia," Ethiopian Journal of Science and Technology, vol. 10, no. 1, pp. 33-50, 2017, no.1.

[14] Bird Life International, Globally Threatened Birds Indicating Priorities for Action, Cambridge United Kingdom: Bird Life International, Cambridge, UK, 2002, http://www.birdlife.net.

[15] M. Eristu, D. Ejigu, and N. Tassie, "The role of church forests in avian conservation: the case in Takussa District, Northwestern Ethiopia. SINET: Ethiop," Journal of Science, vol. 42, no. 2, pp. 50-59, 2019.

[16] M. R. C. Posa and N. S. Sodhi, "Effects of anthropogenic land use on forest birds and butterflies in Subic Bay, Philippines," Biological Conservation, vol. 129, no. 2, pp. 256-270, 2006.

[17] A. J. Urfil, M. Sen, A. Kalam, and T. Maganathan, "Counting birds in India: methodologies and trends, initiatives, international bird strike committee, Amsterdam," Current Science, vol. 89, pp. 25-27, 2005.

[18] D. Crabtree, "Project proposal for the establishment of Altash national park," Unpublished Data, vol. 9, 1997.

[19] National Meteorological Service Agency, "Weather condition record of Quara Woreda at Gelgu town," National Meteorological Service Agency Office, Bahir Dar, Ethiopia, 2018.

[20] F. White, The Vegetation of Africa; A Descriptive Memoir to Accompany the UNESCO/AETFAT/UNSO Vegetation Map of Africa, UNESCO, Paris, France, 1983.

[21] P. W. Webala, N. O. Oguge, and A. Bekele, "Bat species diversity and distribution in three vegetation communities of Meru National Park, Kenya," African Journal of Ecology, vol. 42, no. 3, pp. 171-179, 2004.

[22] W. J. Sutherland, Ecological Census Techniques: A Handbook, Cambridge University Press, Cambridge, UK, 2nd edition, 1996.
[23] C. J. Bibby, N. D. Burgess, and D. A. Hill, Bird Census Techniques, Academic Press, London, UK, 2nd edition, 2000.

[24] S. T. Buckland, D. R. Anderson, J. L. Burnham, and D. L. Borchers, "s," Introduction to distance sampling: estimating abundance of biological population, Oxford University Press, Oxford, England, 2001.

[25] K. Esayas and A. Bekele, "Species composition, relative abundance, and distribution of the avian fauna of the Entoto Natural Park and Escarpment," SINET: Ethiopian Journal of Science, vol. 34, no. 2, pp. 112-122, 2011.

[26] E. Tessfa, D. Ejigu, G. Degife, and N. Tassie, "Diversity, relative abundance, and habitat association of avian species in Tara Gedam Monastery forest and adjacent habitats, Northwestern Ethiopia," Ethiopian Journal of Science and Technology, vol. 13, no. 1, pp. 65-80, 2020.

[27] S. T. Buckland, "Point-transect surveys for songbirds: robust methodologies," The Auk: Ornithological Advances, vol. 123, no. 2, pp. 345-357, 2006.

[28] M. E. Hostler and M. B. Martin, Florida Monitoring Program: transect method for surveying birds, University of Florida Press, FL, USA, 2006.

[29] R. E. Norvell, F. P. Howe, and J. R. Parrish, "A seven-year comparison of relative-abundance and distance-sampling methods," The Auk: Ornithological Advances, vol. 120, no. 4, pp. 1013-1028, 2003.

[30] A. Bekele and S. Aynalem, "Species composition, relative abundance, and habitat association of the bird fauna of the montane forest of Zegie Peninsula and nearby, Lake Tana, Ethiopia," SINET: Ethiopian Journal of Science, vol. 32, no. 2, pp. 45-56, 2009.

[31] B. V. Perlo, Birds of eastern Africa, Collins illustrated checklist Harper Collins publishers, London, UK, 1995.

[32] N. Redman, T. Stevenson, and J. Fanshawe, Birds of the Horn of Africa, Princeton University Press, Princeton, NJ, USA, 2009.

[33] G. Mengesha, "The diversity, distribution, relative abundance, and habitats association of avian fauna of Altash," International Journal of Ecology \& Environmental Sciences, vol. 34, no. 2, pp. 215-222, 2008.

[34] T. Desalegn, "Species diversity and abundance of birds of Addis Ababa Bole international airport Addis Ababa city, Ethiopia," , Addis Ababa University67 pages, Ethiopia, 2011, , M.Sc. Thesis.

[35] D. Pomeroy, Counting Birds. A Guide to Assessing Numbers, Biomass, and Diversity of Afro- Tropical Birds, African Wildlife Foundation, Nairobi, Africa, 1992.

[36] A. Nabaneeta and A. Gupta, "Avian community analysis in fragmented landscapes of cachar district," Journal of Science and Technology, vol. 5, pp. 75-84, 2010.

[37] E. M. Brook and T. Berkead, The Cambridge Encyclopaedia of Ornithology, Cambridge University Press, Cambridge, England, 1991.

[38] P. G. Sreekumer and M. Balakrishnan, "Habitat and altitude preferences of butterflies in Aralam wildlife sanctuary Kerala," Tropical Ecology, vol. 42, pp. 277-281, 2001.

[39] P. Laiolo, F. Dondero, E. Ciliento, and A. Rolando, "Consequences of pastoral abandonment for the structure and diversity of the alpine avifauna," Journal of Applied Ecology, vol. 41, no. 2, pp. 294-304, 2004.

[40] T. G. Martin and H. P. Possingham, "Predicting the impact of livestock grazing on birds using foraging height data," Journal of Applied Ecology, vol. 42, no. 2, pp. 400-408, 2005. 
[41] A. Aich and S. K. Mukhopadhyay, "Comparison of avifauna at the edge of contrasting forest patches in western Ghat hills of India," Ring, vol. 30, no. 1, pp. 5-14, 2008.

[42] D. P. Tubelis and R. B. Cavaicanti, "Community similarity and abundance of bird species in open habitats of a central Brazilian cettado," Orintholgia Neotropical, vol. 12, pp. 57-73, 2001.

[43] P. G. Ryan and A. O. Owino, Habitat association of papyrus specialist birds at three papyrus Uganda, Rwanda, and Burundi, T and A D poyser ltd, London, UK, 2006.

[44] P.-Y. Lee and J. T. Rotenberry, "Relationships between bird species and tree species assemblages in forested habitats of eastern North America," Journal of Biogeography, vol. 32, no. 7, pp. 1139-1150, 2005.

[45] F. C. Estades, "Bird-habitat relationships in vegetation gradient in the Andes of central Chile," The Condor: Ornithological Applications, vol. 99, pp. 719-727, 1997.

[46] W. M. Block and L. A. Brennan, "The habitat concept in ornithology," Current Ornithology, vol. 11, pp. 35-91, 1993.

[47] M. Molla, D. Ejigu, and Y. Yitayih, "Species composition, relative abundance, and habitat association of avifauna in zegie peninsula forest patches and associated wetlands, Bahir dar, Ethiopia," International Journal of zoology, vol. 2021, pp. 1-12, Article ID 9928284, 2021. 